\title{
Adverse drug reaction
}

\section{Ciprofloxacin-induced bone marrow depression}

\author{
Tarun Kumar Dutta, Bhawana A Badhe
}

\begin{abstract}
Summary
Ciprofloxacin, a broad-spectrum fluoroquinolone antibacterial agent, is generally considered to be a safe drug. However, occasionally it may have life-threatening complications. Two instances of bone marrow failure following use of ciprofloxacin are reported. In one case, the bone marrow reverted to normal following withdrawal of the drug. In the other case, the patient succumbed to irreversible bone marrow depression leading to severe thrombocytopenia and uncontrolled bleeding. This could have been an idiosyncratic reaction.
\end{abstract}

Keywords: ciprofloxacin; bone marrow depression; adverse drug reaction

Ciprofloxacin, a drug belonging to the quinolone group of antibiotics, is routinely used for treatment of typhoid fever in endemic areas following the widespread development of chloramphenicol-resistant typhoid fever in these areas. The advantages have been the overall safety and tolerance profile of the drug and scarce reports of resistance. ${ }^{1}$ Nevertheless, it now appears that the use of this drug may occasionally be life-threatening. We present two instances of use of ciprofloxacin in suspected cases of enteric fever (later proved to be non-typhoid cases) associated with lifethreatening bone marrow depression and pancytopenia. In one instance the bone marrow depression reverted to normal with stoppage of ciprofloxacin therapy, in the other instance, Postgraduate Medical Education and Research, Pondicherry 605006 , India

T K Dutta B A Badhe

Accepted 18 February 1999 however, the patient succumbed to the bone marrow depression.

\section{Case reports}

\section{Case 1}

A 24-year-old man, a resident doctor of this institute, was admitted on 25 August 1996 with high-grade fever and chills and rigors for 4 days and jaundice for one day. On examination the patient was found to be febrile and icteric. There were no other positive physical findings. A provisional diagnosis of malaria or viral hepatitis was made. However, the patient had been fully immunised against hepatitis B infection, and examination of his blood failed to reveal malaria parasite. He was empirically given chloroquine, but the fever did not subside and he was started on $500 \mathrm{mg}$ of ciprofloxacin (Minopharm Laboratories, Hyderabad, India) orally bid on the third day of his hospital stay with a fresh provisional diagnosis of typhoid hepatitis. A haemogram done before starting ciprofloxacin showed haemoglobin $9.4 \mathrm{~g} / \mathrm{dl}$, total leucocyte count (TLC) $8.7 \times$ $10 \%$, differential count of neutrophils $87 \%$, eosinophils $1 \%$, basophils $0 \%$, lymphocytes $12 \%$, monocytes $0 \%$, platelets $181 \times 10^{9} / 1$ and reticulocytes $0.3 \%$ (table 1 ). Blood tests 2 days after starting ciprofloxacin were negative for typhoid fever, HIV infection, and HBsAg. Urine examination for leptospira was also negative. A chest X-ray revealed a pneumonic patch in the right upper lobe. His liver function test showed a total bilirubin of $10 \mathrm{mg} / \mathrm{dl}$, direct $3.1 \mathrm{mg} / \mathrm{dl}$. Urine was positive for bile pigments. Crystalline penicillin was added to the treatment in view of the possibility of pneumonia. A repeat haemogram 2 days after starting ciprofloxacin revealed haemoglobin $8.9 \mathrm{~g} / \mathrm{dl}$, TLC $2.1 \times 10^{9} / 1$ and a differential of neutrophils $48 \%$, lymphocytes $36 \%$, eosinophils $16 \%$, platelets $167 \times 10^{9} / 1$ and reticulocytes $0.1 \%$. Three days later (1 September 1996), his haemogram showed further deterioration (table 1) and a peripheral smear revealed pancytopenia. Presuming that the pancytopenia was drug related and since the patient had become afebrile and his jaundice had subsided, both drugs were stopped. His blood counts continued to be low, however, and he developed gingival bleed and purpuric spots on the right cubital fossa, left forearm and thigh over the next week. Examination of the patient's bone marrow revealed hypocellularity with increased lymphocyte count suggesting bone marrow failure (table 2). He was given two units of blood ( $350 \mathrm{ml} / \mathrm{unit}$ ) and four bags of platelet concentrate during this period.
Abbreviations: TLC: total leucocyte count; DC: differential count; N: neutrophils, E: eosinophils, L: lymphocytes, M: monocytes, MC: myelocytes, MMC: metamyelocyte, St: stab form; *: not done 
Haemogram on 7 September 1996 revealed haemoglobin $9.7 \mathrm{~g} / \mathrm{dl}$, TLC $1.5 \times 10^{9} / 1$, platelets $51 \times 10^{9} / 1$ and reticulocytes $0.5 \%$. However, thereafter the blood counts started rising and the haemogram 7 days after stopping ciprofloxacin showed haemoglobin $10.6 \mathrm{~g} / \mathrm{dl}$, TLC $2.9 \times 10^{9} / 1$ and platelets $113.1 \times 10^{9} / 1$. The haemogram became totally normal by 15 September 1996 (see table 1). He became totally asymptomatic and recommenced work in this institute. As the blood counts fell soon after starting ciprofloxacin, the pancytopenia and bone marrow depression were attributed to ciprofloxacin (repeat bone marrow examination to confirm recovery was not done for ethical reasons, although it was clear that the recovery was complete).

Case 2

A 25-year-old man was admitted with a history of high-grade fever and chills for 3 weeks, melaena for 5 days, and bleeding gums, haematuria, haematochezia and purpuric rashes for 3 days. Prior to entry to this institute, he had been treated as a case of malaria with chloroquine; in the absence of a response, he was treated for enteric fever/septicaemia in a private nursing home (10 days before entry) where he was given injections of ciprofloxacin (Cadila Healthcare, Ahmedabad, India), initially for 3 days, then gentamicin, metronidazole, and procaine penicillin for 7 days (cefoperazone was also added on the last four days).

At the onset of illness, his blood counts had revealed a haemoglobin of $12 \mathrm{~g} / \mathrm{dl}$, TLC $9.2 \times$ $10 \%$, differential count of neutrophils $52 \%$, lymphocytes $47 \%$ and monocytes $1 \%$. Peripheral smear had revealed a normal blood picture. His blood cultures were twice sterile. He had had the first episode of bleeding in the form of melaena after 5 days on ciprofloxacin. His total leucocyte count had fallen to $3.2 \times 10^{9}$ $/ 1$ after 8 days on ciprofloxacin ( 2 days before entry to this hospital) and bleeding time was found to be prolonged ( $>10 \mathrm{~min})$. His chest $\mathrm{X}$-ray was normal.

On admission to this institute, the patient had severe pallor, bleeding gums, mild hepatomegaly and purpuric spots all over his body. His haemogram revealed haemoglobin $6.5 \mathrm{~g} / \mathrm{dl}$, TLC $0.4 \times 10^{9} / 1$, a differential count of neutrophils $20 \%$, lymphocytes $80 \%$; platelet count was $7 \times 10^{9} / 1$, and reticulocytes $0 \%$. Peripheral smear revealed severe pancytopenia. His blood was negative for fibrin degradation product. His peripheral smear was negative for malaria parasite. Bone marrow examination showed a hypocellular marrow with predominantly erythrocytic suppression (myeloid:erythroid ratio 6.5) (table 2) and megakaryocytic suppression (? idiosyncratic to ciprofloxacin). Ultrasonogram revealed an organised blood clot in the urinary bladder. Widal test was not suggestive of enteric fever. The initial diagnosis was drug-induced hypoplastic anaemia (since enteric fever as a cause of hypoplastic marrow had been ruled out). The original illness appeared to have been septicaemia.

He was commenced on cephalosporins and aminoglycosides, but despite repeated fresh
Table 2 Bone marrow differential counts of the two patients

\begin{tabular}{llll}
\hline & \multicolumn{2}{c}{ Differential (\%) } & Normal range ${ }^{2}$ \\
\cline { 2 - 3 } Cell type & Case 1 & Case 2 & $(\%)$ \\
\hline Myeloblast & 0 & 1 & $0.3-5.0$ \\
Promyelocyte & - & 4 & $1-8$ \\
Myelocyte & 28 & 25 & $8-16$ \\
Metamyelocyte + stab form & 7 & 25 & $18-40$ \\
Segmented neutrophil & 10 & 4 & $3-11$ \\
Eosinophil & 1 & 1 & $1-5$ \\
Normoblast & 18 & 9 & $18-36$ \\
Lymphocyte & 33 & 26 & $11-23$ \\
Monocyte & 1 & 0 & $0-0.8$ \\
Plasma cell & 2 & 5 & $0.4-3.9$ \\
Myeloid:erythroid ratio & 2.5 & 6.5 & $1.5-3.3$ \\
\hline
\end{tabular}

blood transfusions continued to bleed from various sites and died 2 days after admission.

\section{Discussion}

Ciprofloxacin is generally considered to be a safe and well-tolerated drug. ${ }^{3}$ Its side-effects are mild and not life-threatening, significant side-effects being seen in $<1 \%$ cases. $^{3}$ All sideeffects are reversible with discontinuation of the drug. Haematological adverse effects in particular are usually mild and are seen in only $0.9-1.8 \%$ of cases. ${ }^{45}$ Thus, the drug is recommended for febrile neutropenias. ${ }^{3}$ No bleeding or coagulation abnormality has been noted in the past with this drug. ${ }^{6}$ Reported haematological side-effects include reversible leucopenia ${ }^{7}$ and asymptomatic increase or decrease of platelet count. ${ }^{48}$ There have been reports of haematological side-effects from an Indian study by Karande and Kshirsagar, who reported them in $3.8 \%$ of cases $^{9}$; however, Grover, in another study, reported no haematologic abnormality in any of his patients. ${ }^{10}$ Rare haematological side-effects include transiently acquired Von Willebrand syndrome (reported in two cases). ${ }^{11}$ In another report, asymptomatic prolongation of bleeding time was noted in one patient, but did not occur on re-challenge. ${ }^{6}$ Bone marrow depression has not previously been reported to our knowledge. ${ }^{45}$

In the first case, although the provisional diagnosis was typhoid fever, this was subsequently excluded. Thus, the bone marrow depression could not be attributed to disease per se. The only drug used before the onset of pancytopenia, apart from ciprofloxacin, was chloroquine, which is also not known to cause bone marrow depression. ${ }^{12}$ Even though the patient recovered completely with discontinu-

Learning points
- ciprofloxacin is widely used in typhoid endemic
areas
though safe, it may rarely cause pancytopenia
and bone marrow depression
ciprofloxacin-induced bone marrow depression
should be suspected when blood counts fall soon
after starting ciprofloxacin


ance of ciprofloxacin, re-challenge with the drug was not performed for ethical reasons. In the second case, counts were normal at the onset of illness, and typhoid was ruled out on the basis of the blood report. Since the other drugs used in this patient are not known to cause bone marrow depression, the bone marrow failure was also attributed to ciprofloxacin.

The rapid depression of bone marrow following use of ciprofloxacin suggests an

1 Rodrigues C, Mehta A, Andrews R, Joshi VR. Clinical resistance to ciprofloxacin in salmonella typhi. $\mathcal{F}$ Assoc Physicians India 1998;46:323-4

2 Mazza JG, ed. Manual of clinical hematology. Boston: Little, Brown and Company, 2nd edn, 1995; pp 411-41.

3 Davis R, Markham A, Balfour JA. Ciprofloxacin - an 3 Davis R, Markham A, Balfour JA. Ciprofloxacin - an
updated review of its pharmacology, therapeutic efficacy updated review of its pharmacology, ther

4 Schacht P, Arcieri G, Hullmann R. Safety of oral ciprofloxacin. An update based on clinical trial results. $A m \mathcal{F}$ Med 1989;87(5A):98S-102S

5 Arcieri GM, Becker N, Esposito B, et al. Safety of intravenous ciprofloxacin. A review. Am f Med 1989;87(5A): 92S-97S

6 Pilmore HL, Walker RJ. Prolonged bleeding time during ciprofloxacin therapy. F Clin Pharm Ther 1995;20:45-6.

7 Choo PW, Gantz NM. Reversible leukopenia related to ciprofloxacin therapy. South Med F 1990;83:597-8. idiosyncratic reaction. In one case, pancytopenia was observed only 2 days after use of ciprofloxacin, while in the other case, bleeding symptoms started 5 days after starting ciprofloxacin (even though the drug was stopped on the fourth day). Thus, it is possible that a single dose may cause bone marrow depression in rare instances. Further controlled trials need to be undertaken to confirm this reaction, especially in non-typhoid cases.

8 Jick SS, Jick H, Dean AD. A follow-up safety study of ciprofloxacin users. Pharmacotherapy 1993;13:461-4.

9 Karande SC, Kshirsagar NA. Adverse drug reaction monitoring of ciprofloxacin in pediatric practice. Indian Pediatr 1992;29:181-8.

10 Grover JK. Unwanted effects of ciprofloxacin in Indian population. Indian f Physiol Pharmacol 1993;37:232-4.

1 Castaman G, Lattuada A, Mannuccii PM, Rodeghiero F. Characterisation of two cases of acquired transitory von Willebrand syndrome with ciprofloxacin: evidence for heightened proteolysis of von Willebrand factor. $\mathrm{Am} \mathcal{F}$ Hematol 1995;49:83-6.

12 Webster LT, Jr. Drugs used in the chemotherapy of protozoal infections: malaria. In: Gilman AG, Rall TW, Nies AS, Taylor P, eds. Goodman and Gilman's The pharmacological basis of therapeutics. Singapore: Maxwell Macmillan Publishing Singapore Pvt Ltd, 8th edn. (Maxwell Macmillan International Edition), 1991; pp 978-98. 